\title{
Evaluation of the Tendencies of Urology Residents in Different Training Centers in Turkey on the Use of Fluoroscopy in Operations
}

\author{
Samet Senel ${ }^{1}$, Fatih Sandikci ${ }^{2}$, Ali Yasin Ozercan ${ }^{1}$, Emin Gurtan ${ }^{3}$, Salih Zeki Sonmez ${ }^{4}$, and \\ Hüseyin Cihan Demirel ${ }^{5}$ \\ ${ }^{1}$ Ankara City Hospital \\ ${ }^{2}$ Ankara Diskapi Yildirim Beyzat Training and Research Hospital \\ ${ }^{3}$ Yozgat Bozok University \\ ${ }^{4}$ Istanbul Bagcilar Training and Research Hospital \\ ${ }^{5}$ Sisli Hamidiye Etfal Training and Research Hospital
}

June 23, 2021

\begin{abstract}
Aim: To evaluate the tendency, knowledge, awareness and behavior patterns of urology residents training at different institutions in Turkey about the use of fluoroscopy in operations. Methods: The 13-questioned survey prepared using "Google Forms (C)" as of 01.03.2021 was shared for four weeks in the "WhatsApp@" application group, which includes 279 urology residents studying with university hospitals and training and research hospitals in Turkey. One hundred and thirteen participants, who completed the questionnaire were included in the study. Results: Of the 113 urology residents included in the study, 56 (49.6\%) were studying in university hospitals and $57(50.4 \%)$ were in training and research hospitals. $67.3 \%$ of the residents stated that they never hesitated to participate in the operations which fluoroscopy was used. Additionally, the residents stated that, also $43.4 \%$ of the auxiliary healthcare staff frequently refrain from being involved in these cases $(\mathrm{p}<0.001)$. While $21(37.5 \%)$ of the residents trained in the university hospital reported that they hesitated from these cases, this rate was found that 16 (28.2\%) of the residents who were trained in the training and research hospitals and a significant difference was observed between two groups $(\mathrm{p}<0.016)$. Among residents, the rate of using radioprotective lead apron was $94.7 \%$, and the rate of thyroid shield use was $98.2 \%$. While the rate of using radiation protective glasses was $1.8 \%$, it was learned that none of the residents used radioprotective gloves. Only $5.3 \%$ of the residents stated that they are trained in subjects about the harmful radiation effect. Conclusion: Urology residents in Turkey do not receive sufficient training on the harmful effects of fluoroscopy, which they frequently use in their daily practice. In addition, the residents whom training in university hospitals are more scared of the radiation exposure from fluoroscopy than their colleagues working in training and research hospitals.
\end{abstract}

\section{Evaluation of the Tendencies of Urology Residents in Different Training Centers in Turkey on the Use of Fluoroscopy in Operations}

\section{Abstract}

Aim: To evaluate the tendency, knowledge, awareness and behavior patterns of urology residents training at different institutions in Turkey about the use of fluoroscopy in operations.

Methods: The 13-questioned survey prepared using "Google Forms(C)" as of 01.03.2021 was shared for four weeks in the "WhatsAppß" application group, which includes 279 urology residents studying with university hospitals and training and research hospitals in Turkey. One hundred and thirteen participants, who completed the questionnaire were included in the study. 
Results: Of the 113 urology residents included in the study, 56 (49.6\%) were studying in university hospitals and $57(50.4 \%)$ were in training and research hospitals. $67.3 \%$ of the residents stated that they never hesitated to participate in the operations which fluoroscopy was used. Additionally, the residents stated that, also $43.4 \%$ of the auxiliary healthcare staff frequently refrain from being involved in these cases $(\mathrm{p}<0.001)$.

While $21(37.5 \%)$ of the residents trained in the university hospital reported that they hesitated from these cases, this rate was found that $16(28.2 \%)$ of the residents who were trained in the training and research hospitals and a significant difference was observed between two groups $(\mathrm{p}<0.016)$. Among residents, the rate of using radioprotective lead apron was $94.7 \%$, and the rate of thyroid shield use was $98.2 \%$. While the rate of using radiation protective glasses was $1.8 \%$, it was learned that none of the residents used radioprotective gloves. Only $5.3 \%$ of the residents stated that they are trained in subjects about the harmful radiation effect.

Conclusion: Urology residents in Turkey do not receive sufficient training on the harmful effects of fluoroscopy, which they frequently use in their daily practice. In addition, the residents whom training in university hospitals are more scared of the radiation exposure from fluoroscopy than their colleagues working in training and research hospitals.

Keywords: Fluoroscopy; Radiation; Residents; Survey; Training centers

\section{What is known?}

- Percutaneous nephrolithotomy is the most preferred operation by urology residents for fluoroscopy.

- Urology residents do not receive sufficient formal training on the harmful effects of fluoroscopy and prevention methods, which they frequently use in urology practice.

- Urology residents do not take adequate precautions against the harmful effects of radiation in cases where fluoroscopy is used.

\section{What our study adds?}

- The tendency of urology residents to use fluoroscopy in cases varies according to the institution which they training in.

- Urology residents do not prefer to use too much fluoroscopy in an operation (even if the operation can be completed in a single session). Instead, they tend to afford more sessions for one operation. In Turkey, this trend is more noticeable in urology residents working in university hospitals than their colleagues working in training and research hospitals.

- It is also noticeable that in Turkey, urology residents in university hospitals are more wary of the harmful effects of fluoroscopy than their colleagues working in training and research hospitals.

\section{Introduction}

With the advances in technology, open surgical interventions have decreased in modern urology practice and the use of endourological procedures has increased. ${ }^{1}$ Frequently used endourological procedures such as percutaneous nephrolithotomy, endoscopic ureter stone treatments, and retrograde intrarenal operations are mostly performed under fluoroscopy guidance. During these fluoroscopic-guided procedures, surgeons, patients and operating room staff are exposed to a significant amount of ionizing radiation. ${ }^{2}$

Stochastic (mutation and cancer) and deterministic effects may occur as a result of radiation exposure. This effect is related to the duration, dose and protection used. ${ }^{1}$ For this reason, the International Radiation Commission recommends that the radiation dose exposed should not exceed an average of $20 \mathrm{mSv}$ (milisievert)/year in a five years period. ${ }^{3}$

In order to avoid these negative effects of radiation, personal protective equipment such as lead apron, thyroid shield, radiation protection gloves and goggles should be used and basic principles should be observed. ${ }^{1}$ In addition, it is necessary to use a dosimeter to determine the cumulative radiation dose exposed. ${ }^{6}$ However, studies on this subject have revealed that protective equipment and dosimeters are not used enough among urologists, and there is a lack of knowledge and awareness on this subject. ${ }^{2,6,7}$ 
Urology residency is an important occupational group with a high risk of exposure to radiation. In Turkey, urology residency training is applied in university hospitals and training and research hospitals. Although there are studies evaluating the awareness of radiation exposure among the urology residents, there is no study comparing the tendency to use fluoroscopy in different institutions in the literature. In this study, we evaluated the tendency, knowledge, awareness and behavior patterns of urology residents in Turkey regarding the use of fluoroscopy in different institutions.

\section{Materials and Methods}

Ethics committee approval was obtained from the local ethics committee for the survey study (Approval number: E2-21-502). Participants were informed that the data will be used for scientific purposes only. Physicians working as urology resident in university hospitals and training and research hospitals in Turkey were included in the study. The 13-questioned survey was prepared via "Google Forms@)". The questionnaire form was shared once every two days for 4 weeks as of 01.03 .2021 via "WhatsApp@" application, which includes 279 urology residents. During this period, 113 urology residents who participated in the questionnaire were included in the study. The answers given by the participants were kept confidential.

The survey included the questions that; the range of participants ages, the year of urology residency, the institution (university hospital or training and research hospital) and the surgical techniques using fluoroscopy during their training. In addition, the participants were also questioned about their own and auxiliary healthcare staff tendency to refrain from surgeries using fluoroscopy and to replace a surgery requiring fluoroscopy with a non-fluoroscopy method. It was evaluated whether they and their auxiliary healthcare staff received training on dosimeter usage, radiation dose of fluoroscopy and protection methods. Their opinions on the protective equipments and adequacies and regular controls of them and their knowledge of ionizing radiation protection methods were questioned also.

\section{Statistical analysis}

Statistical data analysis was performed using SPSS version 20.0. Numerical data; It was expressed as the number of participants and the percentage [n (\%)]. Chi-square and Fisher's Exact test were used to compare categorical data. $\mathrm{P}<0.05$ was considered statistically significant.

\section{Results}

One hundred and thirteen participant urology residents completed the questionnaire. Of the residents, 56 $(49.6 \%)$ were training in university hospitals and 57 (50.4\%) were in training and research hospitals. Eightyone $(71.7 \%)$ of the residents were between the ages of $25-30$ and $60(53.1 \%)$ were in 1-3 years of their education. One hundred and twelve $(99.1 \%)$ of the participants reported that they preferred to use fluoroscopy in percutaneous nephrolithotomy (PNL), 75 (66.4\%) of them preferred in retrograde intrarenal surgery (RIRS) and $71(62.8 \%)$ preferred in nephrostomy/double $\mathrm{J}(\mathrm{DJ})$ insertion operations.

In the light of the answers given by the participants, it was revealed that $67.3 \%$ of the residents never hesitated to enter the cases requiring fluoroscopy, while it was learned that $43.4 \%$ of the auxiliary healthcare staff often avoided these cases. According to this data, a statistically significant difference was found between the two healthcare worker groups $(\mathrm{p}<0.001)$. In addition, when the participants were divided into two groups according to the institution they training in, $21(37.5 \%)$ of the residents in university hospitals reported that they hesitated to participate in operations that fluoroscopy was used, while $16(28.2 \%)$ of the residents in training and research hospitals stated that they had same anxiety. This difference was also found to be statistically significant too $(\mathrm{p}=0.016)$.

According to the answers, $39(69.6 \%)$ of the residents in university hospitals stated that they preferred methods (even if it requires more sessions of operations) in which they would not use fluoroscopy at different frequencies, while in training and research hospitals this number was $33(57.9 \%)$, and the difference was found to be statistically significant $(\mathrm{p}=0.042)$.

It was concluded in the study that, $68.1 \%$ of the residents and auxiliary healthcare staff did not use dosime- 
ters in any operation which performed with fluoroscopy. Also obtained from this study that, only six of the residents (5.3\%) received training about the harmful radiation effect of fluoroscopy, radiation protection method, etc. According to the answers of questions about the necessary measures to reduce the effect of ionizing radiation, 107 of the participants $(94.7 \%)$ reported that they used lead aprons in cases where fluoroscopy was used, $111(98.2 \%)$ of them used thyroid shields, while only two (1.8\%) of them used radioprotective glasses reported that they use. It was learned that none of the residents was using the radioprotective gloves.

Finally, 106 of the residents (98.2\%) answered "no" to the question of "Do you think that protective equipment is regularly checked for effectiveness?". And, 109 (96.5\%) residents answered as "I don't know/I don't do" the question of "Do you know / do you perform, what should be done to reduce the harmful radiation effect after fluoroscopy?" The data about the answers given by the residents to the survey are shown in Table 1.

\section{Discussion}

In Turkey, students who complete the formal six-years medical school education receive the title of medical doctor. They are then subjected to a nationwide central examination to be able to receive specialist training in a branch. Those who are successful in the exams receive training their residency in university hospitals or training and research hospitals for a period of time that varies according to each department. In our study, which evaluated the tendency of urology residents studying in different institutions in Turkey to use fluoroscopy in urological operations; interestingly, it was concluded that, even though all of them are at similar education levels on radiation exposure, the residents training in university hospitals had more anxiety about using fluoroscopy, than their colleagues in training and research hospitals. Similarly, it is seen that unlike their colleagues in training and research hospitals, the residents in university hospitals prefer to operate a case with a different method without using fluoroscopy in more sessions, than a single session with using fluoroscopy. The reason for this may be that the residents in university hospitals have higher awareness of the harmful effects of ionizing radiation exposure under the mentorship of senior urologists who provide medical education as well as their specialty education. In addition, it is seen that auxiliary healthcare staff much more avoid the cases in which fluoroscopy is used than urology residents. This can be explained by the fact that residents with concern of training in their occupation, ignoring ionizing radiation exposure. There are many studies in the literature about the awareness of urology residents to radiation exposure. ${ }^{1,6,8-10}$ However, this is the first study to evaluate residents' tendencies to use fluoroscopy according to their educational institutions.

Ionizing radiation is an serious health problem, faced by practitioners when they apply it during medical diagnosis and treatment. While applying these procedures, the harmful effects of radiation must be taken into account. Especially in recent years, the increase in endourological interventions and the parallel increase in exposure to radiation impose an important responsibility on urologists in order to protect themselves, auxiliary healthcare staff and their patients. ${ }^{11}$ Radiation exposure causes serious morbidity and mortality. While causing cellular damage with its cumulative effect, it causes leukemia, lymphoma, thyroid cancer, bladder cancer, liver cancer, esophageal cancer, breast cancer, skin cancer, cataracts and central nervous system cancers and also it has a deterministic effect by causing cell death at high doses. ${ }^{6}$ For this purpose, the use of a lead apron, thyroid shield, radiation protective glasses and gloves is recommended in cases where fluoroscopy is used, and it is known that these protective equipment prevent the harmful effects of ionizing radiation. ${ }^{1}$ However, many studies have shown that most urologists do not have sufficient knowledge about the harmful effects of radiation and do not take adequate precautions against radiation. ${ }^{1,4,12}$ In a study which Harris et al. evaluated 136 urology residents in the United States, it was shown that almost half of the residents did not receive a formal radiation safety training. In the same study, it was reported that $99 \%$ of the residents used a thyroid shield, $97 \%$ of them regularly wore a lead apron, but only $9 \%$ of the residents used radiation protective glasses. Besides, it has been shown that none of the assistants wore radiation protective gloves. ${ }^{6}$ In another survey study conducted on urology residents in Canada, it was stated that although the rate of thyroid shield use was $96 \%$ but $24 \%$ of them used it irregularly. While the rate of use of radiation protective lead apron is $13 \%$, it has been shown that almost no residents use radiation protective gloves. In this study, it was also emphasized that $70 \%$ of the residents did not use dosimetry. ${ }^{10}$ Similar results are 
also observed in studies conducted in Europe. ${ }^{9}$ In our study, it is seen that while the use of lead aprons and thyroid shields is quite high among urology residents in Turkey, almost none of the residents use radiation protective glasses and gloves. Additionally, $68.1 \%$ of the residents never used dosimetry and only $5.3 \%$ stated that they received training on radiation safety. Another striking result is that the vast majority of urology residents do not believe that protective equipment is regularly checked for effectiveness.

Fluoroscopy is used in many operations in urology. In the study conducted by Altintas et al., $53.9 \%$ of the participants answered the question "In which case do you need fluoroscopy device the most" as PNL. ${ }^{8}$ In our study, it is also understood that fluoroscopy is almost used in all PNL cases. In operations of RIRS and nephrostomy/DJ insertion, fluoroscopy was preferred in one of three patients. We think that this difference may have arisen due to studies showing the effectiveness and reliability of the non-fluoroscopic RIRS technique. ${ }^{13}$

There are some limitations of our study. First of all, this study is a survey-based study and only those who preferred to participate in the survey via "WhatsApp@)" were included in the study. Apart from this, the low number of participants is another handicap. In addition, the status of the auxiliary healthcare staff to avoid cases using fluoroscopy was evaluated according to their responses to urology residents. Nevertheless, it is the first study to be conducted due to the tendencies of urology residents training in different institutions in Turkey about the use of fluoroscopy and making a social evaluation from a different perspective makes our study valuable.

\section{Conclusion}

Urology residents in Turkey are refraining from being involved in operations where fluoroscopy is used. Urology residents training in university hospitals are more avoid of radiation exposure than their colleagues in training and research hospitals and tend to apply these operations with methods that do not use fluoroscopy whenever possible. The awareness of urology residents (especially the residents in training and research hospitals) about the effects of radiation exposure and methods of protection should be increased.

\section{Conflict of interest}

None of the authors received any type of financial or nonfinancial support that could be considered a potential conflict of interest regarding the manuscript or its submission.

\section{Funding}

No funding sources.

\section{Author contribution}

Dr SS had the primary responsibility for writing the manuscript. Dr SS, Dr FS and Dr AYO participated in protocol development, preliminary data analysis. Dr EG, Dr SZS and Dr HCD participated in the development of the protocol and analytical framework for the study and the revision of the manuscript.

\section{Data availability statement}

The data that support the findings of this study are openly available in Figshare Repository at https://figshare.com/s/c0c534a6d5b61f15ec51

\section{References}

1. Tok A, Akbas A, Aytan N, Aliskan T, Cicekbilek I, Kaba M, Tepeler A. Are the urology operating room personnel aware about the ionizing radiation? Int Braz J Urol. 2015 Sep-Oct;41(5):982-9. 2. Söylemez H, Altunoluk B, Bozkurt Y, Sancaktutar AA, Penbegül N, Atar M. Radiation exposure-do urologists take it seriously in Turkey? J Urol. 2012 Apr;187(4):1301-5 3. The 2007 Recommendations of the International Commission on Radiological Protection. ICRP publication 103. Ann ICRP. 2007;37(2-4):1-332 4. Brateman L. Radiation safety considerations for diagnostic radiology personnel. Radiographics. 1999 Jul-Aug;19(4):103755. 5. Wang JX, Zhang LA, Li BX, Zhao YC, Wang ZQ, Zhang JY, Aoyama T. Cancer incidence and 
risk estimation among medical x-ray workers in China, 1950-1995. Health Phys. 2002 Apr;82(4):455-66. 6. Harris AM, Loomis J, Hopkins M, Bylund J. Assessment of Radiation Safety Knowledge Among Urology Residents in the United States. J Endourol. 2019 Jun;33(6):492-497. 7. Söylemez H, Sancaktutar AA, Silay MS, Penbegül N, Bozkurt Y, Atar M, Altunoluk B, Bodakci MN, Hatipoglu NK. Knowledge and attitude of European urology residents about ionizing radiation. Urology. 2013 Jan;81(1):30-5. 8. Altintas E, Bahceci T, Batur AF, Kaynar M, Kilic O, Akand M, Goktas S, Gul M. A survey analysis of knowledge levels of urologists about radiation safety and fluoroscopy use. Int J Clin Pract. 2021 Apr;75(4):e13862. 9. Söylemez H, Sancaktutar AA, Silay MS, Penbegül N, Bozkurt Y, Atar M, Altunoluk B, Bodakci MN, Hatipoglu NK. Knowledge and attitude of European urology residents about ionizing radiation. Urology. 2013 Jan;81(1):305. 10. Friedman AA, Ghani KR, Peabody JO, Jackson A, Trinh QD, Elder JS. Radiation safety knowledge and practices among urology residents and fellows: results of a nationwide survey. J Surg Educ. 2013 MarApr;70(2):224-31. 11. Castane W da EG. How to protect yourself and others from radiation. In: Smith AD (ed) Smith's textbook of endourology, 2nd edn. 11-142007. 12. Bagley DH, Cubler-Goodman A. Radiation exposure during ureteroscopy. J Urol. 1990 Dec;144(6):1356-8. 13. Senel C, Tuncel A, Balci M, Asfuroglu A, Aykanat C, Guzel O, Aslan Y. Safety and reliability of fluoroscopy-free technique in retrograde intrarenal surgery. Minerva Urol Nefrol. 2018 Dec;70(6):606-611.

\section{Hosted file}

table.docx available at https://authorea.com/users/383696/articles/527422-evaluation-of-thetendencies-of-urology-residents-in-different-training-centers-in-turkey-on-the-use-of-

fluoroscopy-in-operations 